\title{
The effect of landmark and body-based sensory information on route knowledge
}

\author{
Roy A. Ruddle • Ekaterina Volkova $\cdot$ Betty Mohler • \\ Heinrich H. Bülthoff
}

Published online: 1 December 2010

(C) Psychonomic Society, Inc. 2010

\begin{abstract}
Two experiments investigated the effects of landmarks and body-based information on route knowledge. Participants made four out-and-back journeys along a route, guided only on the first outward trip and with feedback every time an error was made. Experiment 1 used 3-D virtual environments (VEs) with a desktop monitor display, and participants were provided with no supplementary landmarks, only global landmarks, only local landmarks, or both global and local landmarks. Local landmarks significantly reduced the number of errors that participants made, but global landmarks did not. Experiment 2 used a headmounted display; here, participants who physically walked through the VE (translational and rotational body-based information) made $36 \%$ fewer errors than did participants who traveled by physically turning but changing position using a joystick. Overall, the experiments showed that
\end{abstract}

This research was supported by an Alexander von Humboldt Fellowship for Experienced Researchers awarded to R.A.R., as well as by the Max Planck Society and the WCU (World Class University) program through the National Research Foundation of Korea, funded by the Ministry of Education, Science and Technology (R31-2008000-10008-0). We thank Michael Kerger and Michael Weyel for their technical assistance.

R. A. Ruddle $(\bowtie)$

School of Computing, University of Leeds,

Leeds, UK

e-mail: r.a.ruddle@leeds.ac.uk

R. A. Ruddle • E. Volkova • B. Mohler $\cdot$ H. H. Bülthoff

Max Planck Institute for Biological Cybernetics,

Tübingen, Germany

H. H. Bülthoff $(\triangle)$

Department of Brain and Cognitive Engineering,

Korea University,

Seoul, South Korea

e-mail: heinrich.buelthoff@tuebingen.mpg.de participants were less sure of where to turn than which way, and journey direction interacted with sensory information to affect the number and types of errors participants made.

Keywords Navigation - Spatial knowledge - Virtual reality . Locomotion interfaces

One of the primary types of navigation that people perform in everyday life is following routes between specific places-for example, from home to work, to and from the offices of colleagues, and to and from particular shops in their own neighborhood ("National Travel Survey: Why People Travel," 2009). In its most basic form, route knowledge comprises sequences of decisions - for example, "straight on, turn left, and then turn right" (Siegel \& White, 1975). However, to this are added metric information (distances and turn angles), obtained from both internal (body-based) and external sensory sources, and information about features such as landmarks, primarily perceived visually (Lynch, 1960; Montello, 1998). During everyday route-finding, it is estimated that people make an average of one error per week, with $49 \%$ of those occurring when people turn in the wrong place (i.e., travel straight on where they should have turned or turn where they should have continued straight) or turn the wrong way (Williamson \& Barrow, 1994). As compared with basic route knowledge, metric information and feature information make people's knowledge richer, so that they should make fewer errors during navigation.

The present study investigated participants' route knowledge when they made a sequence of out-and-back journeys along a route. Participants were guided on the first outward trip, but subsequently they had to find their own way (feedback was given every time they made an error). Therefore, the participants' task had similarities with the 
everyday scenario of following instructions to a new place (e.g., someone's office) but finding one's own way back, and on subsequent journeys keeping to the original route, because that is the only part of the environment known.

The present study had three main motivations. First, although we live in a world that contains many landmarks and other visual details, little is known about the effect that different types of landmark have on our ability to navigate. To investigate this, in Experiment 1 we used desktop 3-D virtual environments (VEs) and asked participants to navigate routes when no supplementary landmarks, only global landmarks (information visible from anywhere), only local landmarks (information only visible within a locality), or both global and local landmarks were provided.

Second, it is commonly suggested that body-based information (proprioception, etc.) only plays a minor role in large-scale navigation because of the errors that accumulate during path integration (Foo, Warren, Duchon, \& Tarr 2005; Loomis, Klatzky, Golledge, \& Philbeck 1999), and that the translational component of body-based information is particularly unimportant (Klatzky, Loomis, Beall, Chance, \& Golledge 1998). However, this does not explain the difficulty that participants often have navigating VEs (Ruddle \& Lessels, 2009; Ruddle \& Péruch, 2004). Experiment 2 investigated the translational component by asking participants either to physically walk along routes in a $13 \times 12$ m tracking hall that "contained" the VEs or to travel by making physical turns but changing position using a joystick. In both cases, participants viewed the VEs by wearing a head-mounted display (HMD).

Third, very few studies have previously investigated route knowledge when participants traveled both to and from given locations (exceptions are Cornell, Heth, \& Rowat 1992; Hurlebaus, Basten, Mallot, \& Wiener 2008; Ishikawa \& Montello, 2006), despite that being one of the most common navigational tasks we perform in everyday life ("National Travel Survey: Why People Travel," 2009). The cognitive work that is required to transform route knowledge gained when traveling in one direction so that it can be used when the route is reversed depends on what information is contained within that route knowledge. No previous studies have investigated either the role of different categories of landmarks or different components of body-based information when routes are traversed both outward and then back.

This research is important from two perspectives. From a theoretical perspective, this study shows the effects that global versus local landmarks and that translational bodybased information have on route knowledge. The effect on navigational performance was measured by counting the number of errors that participants made, and we classified the errors into specific types to highlight particular deficiencies in participants' mental representations of the routes. Analysis of the behavioral data (the extent to which participants looked around) provided indicators of the external information that participants used to make routefinding decisions. On the other hand, from an applied perspective, VEs have not yet achieved their potential in areas such as spatial training for rescue and rehabilitation (Farrell et al., 2003). This study indicates the potential benefits of physical locomotion devices.

\section{Landmarks}

Landmarks may be classified according to a number of attributes, including visibility (global vs. local), salience, distinctiveness, relevance, reliability, and persistence (Gillner, Weiß, \& Mallot 2008; Stankiewicz \& Kalia, 2007; Steck \& Mallot, 2000). This section briefly reviews research that has investigated the effects of global and local landmarks on navigation and hypothesizes how landmarks improve route knowledge and affect the types of errors that people make.

Global (distal) landmarks always provide orientation information (e.g., the North Star or the sun) and sometimes provide crude positional information (e.g., a radio mast on a hill overlooking a town may be noticeably nearer some parts of that town than others; Steck \& Mallot, 2000). Research has shown that global landmarks only benefit some tasks, because adding a photorealistic surrounding scene had no effect when participants exhaustively searched a virtual room (Ruddle \& Lessels, 2009), but adding distinctive buildings visible from far away to an otherwise homogeneous city significantly improved the accuracy with which participants spatially arranged photographs of a route they had traveled (Evans, Skorpanich, Gärling, Bryant, \& Bresolin 1984). Navigating a route has more similarity to the task presented by Evans et al. than to an exhaustive search. Therefore, we hypothesized that adding global landmarks to an environment would reduce the overall number of errors that participants made when they traversed a route, because they would turn the wrong way less often. By providing a stable, allocentric frame of reference, global landmarks should also allow participants to use the knowledge gained while traversing a route in one direction when returning in the other.

Local (proximal) landmarks always provide positional information and, unless they are symmetrical, also provide orientation information. As with their global counterparts, research has shown that local landmarks also only benefit certain tasks. For example, local landmarks had no effect when participants navigated a virtual building to find targets in any order (Ruddle, 2005), and when the target order was specified, navigation improved only if the landmarks were everyday objects instead of abstract 
patterns (Ruddle, Payne, \& Jones 1997). However, adding object landmarks to an environment halved the number of trials that participants required to learn the shortest route in one direction between two places (Jansen-Osmann \& Fuchs, 2006). Therefore, we hypothesized that adding local landmarks to an environment would also reduce the overall number of errors that participants made when they traversed a route, because they would more often turn in the correct place.

Local landmarks may be either associative or beacons, the former indicating just a decision point's position but the latter indicating both where and which way one should turn (Waller \& Lippa, 2007). Research into effective route directions has indicated that landmarks tend to be used in an associative manner (Denis, 1997), which means that both the sequence in which landmarks are encountered and the actions that are executed at each landmark need to be inverted when a route is reversed. Therefore, we hypothesized that participants would make more errors on return journeys than when traveling in the direction in which a route was initially traversed.

What happens, then, if both global and local landmarks are added to an environment? What little previous research there has been has shown that the accuracy of participants' route choices at decision points was slightly, but significantly, reduced if either type of landmark was unexpectedly removed (Steck \& Mallot, 2000). Other studies have included conditions that had either global or local landmarks. As compared with none, either type of landmark significantly improved the accuracy with which participants spatially arranged photographs of a route they had traveled (Evans et al., 1984), and in unconstrained navigation, global landmarks facilitated the learning of the general direction of a route, whereas local landmarks facilitated the learning of a specific path (Hurlebaus et al., 2008). Given that global landmarks primarily provide orientation information and local landmarks primarily provide position information, we hypothesized that the two types of landmarks would complement each other and reduce the number of errors that participants made relative to the situation in which just one type was present.

\section{Body-based information}

When we travel, body-based cues are provided for the translational and rotational components of movement. This section briefly reviews research that has investigated the effect of either component on navigation, and then we hypothesize how the addition of the translational component may improve route knowledge and affect the errors that participants make relative to a baseline condition that provides visual and rotational body-based information.
There has been much more previous research into the effect of this baseline than into the effect of combined rotational/translational body-based information, because the baseline can be implemented using a commonplace HMD setup, but the translational component requires specialist tracking facilities.

Previous research into the components of body-based information has produced markedly different results. In studies that used the basic spatial task of triangle completion, the rotational component of body-based information was essential if participants were to avoid making large systematic errors, but the translational component was not required (Avraamides, Klatzky, Loomis, \& Golledge 2004; Klatzky et al., 1998). The errors were associated with participants' failure to update their cognitive heading. By contrast, when participants traversed a route in a VE and then pointed to targets that had been encountered, performance improved gradually as more components of body-based information were provided (Chance, Gaunet, Beall, \& Loomis 1998). Participants who physically walked through the VE (translational and rotational body-based information) estimated directions significantly more accurately than participants who were only provided with visual information. Participants who made physical turns but translated using a joystick performed with intermediate accuracy.

A third pattern of results was found in studies where participants had to explore a space, rather than traverse one path. When a room-sized space was exhaustively searched for targets, full (translation and rotation) body-based information produced a step change in performance, because participants completed twice as many trials perfectly as participants who had been provided either with visual and rotational body-based information or with just visual information (Ruddle \& Lessels, 2009). This was supported by other research, which showed that adding only rotational body-based information to visual information had no effect on navigational performance when participants learned the location of places in virtual buildings that were laid out either orthogonally (Ruddle, Payne, \& Jones 1999) or obliquely (Ruddle \& Péruch, 2004).

Differences between the results of the above studies may be explained in terms of task difficulty (see Experiment 2's Discussion). We hypothesized that adding the translational component of body-based information would improve route knowledge, as compared with a baseline condition in which visual information and rotational body-based information were provided, because it would reinforce participants' knowledge of how far they had traveled, and so would help them turn in the correct place. This advantage should persist when a route was reversed, so we hypothesized that the patterns of errors in the baseline and translation conditions would be similar. 


\section{Overview of experiments}

The remainder of this article reports two experiments that investigated route knowledge. The results quantified participants' performance (number of errors), classified the errors into types to highlight deficiencies in the participants' route knowledge, and used changes in participants' view directions to indicate how external information was used to make route-finding decisions. All of the participants were naive, gave informed consent, and were paid an honorarium for their participation. The experiments were approved by the local ethics committee.

Experiment 1 investigated the effect of landmarks by providing participants with no supplementary landmarks, with only global landmarks, with only local landmarks, or with global and local landmarks. Experiment 2 investigated the effect of adding translational bodybased information to a baseline condition that provided visual and rotational body-based information. In both experiments, participants made four out-and-back journeys along a route. We tested hypotheses about how participants' errors on return journeys would be affected by the sensory information provided. Journey number was included as a factor in the analyses to analyze the longitudinal changes that took place in participants' route knowledge.

\section{General Method}

\section{Materials}

Environments Four layouts were designed. Each was a virtual marketplace, which comprised a grid layout of square stalls and one long stall along each edge of the marketplace. The height of each stall was determined randomly $(\min =2.0 \mathrm{~m}, \max =2.9 \mathrm{~m})$, the length and width were both $0.75 \mathrm{~m}$, and the corridor width was also $0.75 \mathrm{~m}$. Each participant used the simplest layout to practice the user interface, a second to practice the task, and one of the final two layouts for the test (see Figs. 1 and 2).

The interface practice layout contained a $5 \times 5$ grid of stalls and a 24-m route, which was marked by green arrows. The environment contained no pictorial cues (every stall was "empty," as in Fig. 2a).

The layout used for the practice task contained a $6 \times 9$ grid of stalls and a $15-\mathrm{m}$ route that had nine decision points (3 straight on, 6 turns). Four versions of the layout were created, in which (1) there were no pictorial cues (no supplementary landmarks), (2) every stall contained a picture of an everyday object that was visible from one side (local landmarks), (3) the four walls along the edges of the marketplace each contained a picture of an everyday

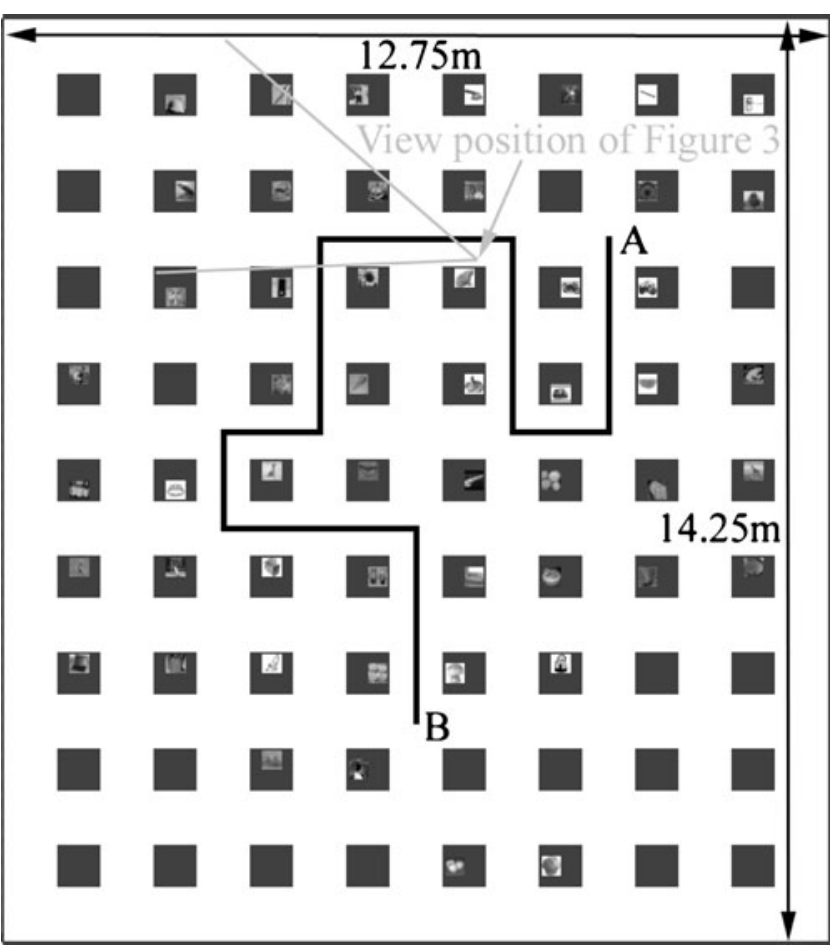

Fig. 1 Plan view of the layout of one of the test routes, showing the pictures used in the Local condition (blank stalls were those where a picture had been allocated to a side that could not be seen from that route). Participants started at $\mathrm{A}$ and did four out-and-back journeys (A-B-A-B-A-B-A-B-A)

object (global landmarks), or (4) the layout contained all the pictures for both the local and global landmarks.

Both layouts used for the test contained an $8 \times 9$ grid of stalls and a $22.5 \mathrm{-m}$ route that had 14 decision points (6 straight on, 8 turns; see Fig. 1). The routes used for the two test layouts had the same overall shape, but the initial start points were at opposite ends of the space, different pictures were used as landmarks, and the local landmarks were placed on different sides of the stalls. As for the practice task, four versions of each layout were created: (a) no landmarks, (b) local landmarks, (c) global landmarks, and (d) local and global landmarks (see Fig. 2).

Landmarks All of the landmarks were constructed from pictures of easily recognizable objects (they were all correctly named in a pilot study that involved 5 participants); they were clearly visible in the environment and remained in fixed locations. Thus, the objects would be considered to be reliable landmarks. All of the landmarks had asymmetries, which provided a certain amount of directional information, as is often the case in the real world.

When present, the local landmarks were positioned so that each stall had a landmark that was visible from one side, through a $0.60-\mathrm{m}$ wide opening in the stall (each landmark was texture mapped onto a horizontal and a vertical surface inside the stall; see Fig. 2). There were 15 

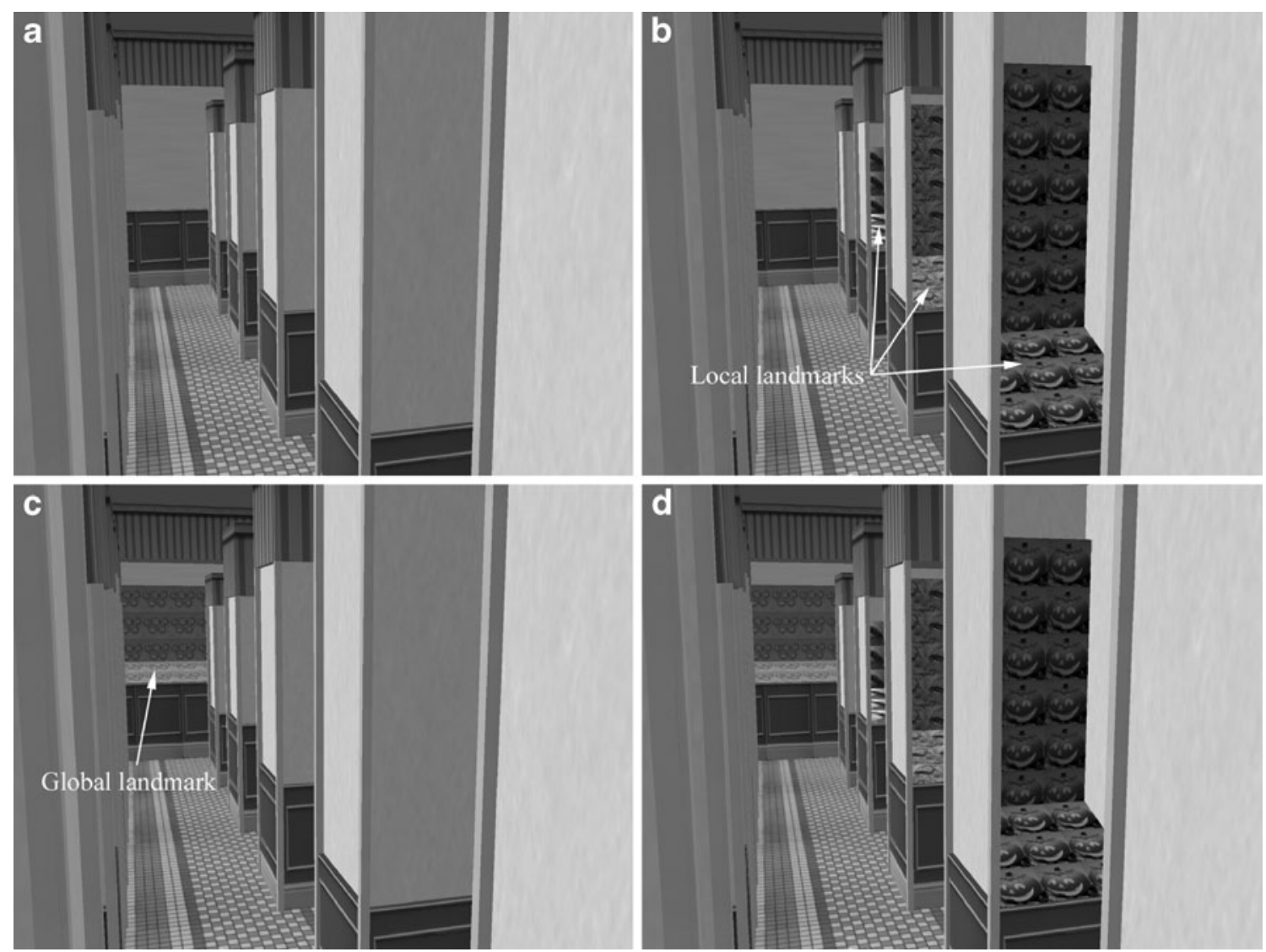

Fig. 2 Views inside the four versions of the layout shown in Fig. 1. The view position and orientation are the same in each example: (a) no supplementary landmarks, (b) local, (c) global, and (d) local and global landmarks

landmarks along the route and another 15 landmarks along decoy path segments (for every turn, the decoy segment went the same distance as the next route segment, but in the opposite direction). The landmark positions for each layout were generated randomly by a computer program.

The pictures used for the global landmarks were texture mapped onto the four walls of the marketplace. Each of these pictures was different, was visible throughout the environment, provided a unique cue for one of the four cardinal directions, and helped participants judge how far they were from a given wall.

Hardware and software The VE software was written using $\mathrm{C}++$. It ran on a Dell Inspiron M1710 laptop (2.16GHz T7400 CPU, 2 GB RAM, NVIDIA GeForce Go 7950 GTX graphics card) and rendered the scene at 60 frames/s. Participants' position and orientation in a VE was recorded at the same rate to a $\log$ file for subsequent analysis by custom-developed postprocessing software.

User interface In Experiment 1, participants used a Logitech Rumblepad, a common interface device for PC gaming, to control their movements along the routes. Manipulating the left joystick allowed participants to travel at a speed of up to $0.9 \mathrm{~m} / \mathrm{s}$ (a slow walk) in any direction (forward, backward, diagonally, etc.), and the right joystick allowed participants to vary the view heading and pitch. The heading and pitch could be changed by up to $120 \% \mathrm{~s}$ and $25 \%$, respectively. Heading changes were seamless, but pitch was constrained so that participants could only look between vertically up and down.

In Experiment 2, participants in the Walk group physically walked around a large tracking hall (see www. cyberneum.org) while viewing the VEs in an HMD. The position and orientation of a participant's head was tracked in six degrees of freedom using a Vicon MX13 motion capture system. Participants in the Rotate group stood in one place and viewed the VE in stereo on the HMD. They achieved movement by physically rotating, tracked by the Vicon system, but using a Rumblepad joystick to translate.

\section{Procedure}

The procedure was divided into four stages: (1) interface practice, (2) practice the task, (3) perform the test, and (4) answer a questionnaire. On average, each participant took $1.5 \mathrm{~h}$ to complete the experiment. 
The interface practice started with the experimenter demonstrating how to traverse the interface practice route, using the desktop display and gamepad interface. Participants then made five out-and-back journeys (A to B, then back to A) along this route. In Experiment 1, all of the journeys were done using the desktop display and gamepad, because those were the display and interface participants would use in the subsequent stages. In Experiment 2, the first journey was done using the desktop display and gamepad, to allow the participant to clarify the procedure face to face with the experimenter, and the other journeys were performed using the HMD.

The practice task was performed using a desktop display (Experiment 1) or HMD (Experiment 2; walk vs. rotate). Participants made four out-and-back journeys along the practice task route, guided by arrows embedded within the VE on the first outward trip, but subsequently having to find their own way. If participants tried to travel down the wrong corridor, a red cross was presented and movement down that corridor was prevented, so that participants were not allowed to deviate from the route. At the start of each trip, participants were always facing the direction in which they needed to travel.

The test adopted the same procedure as the practice task. That is, four out-and-back journeys, guided on the first outward trip, and with error feedback (the red cross) if participants attempted to travel down the wrong corridor, so that participants were not allowed to deviate from the route. The test route was $50 \%$ longer and had more turns than the one used in the practice task.

Experiment 1's questionnaire had seven questions: (1) How often do you use 3-D chat worlds? (2) How often do you play 3-D computer games? (3) How easy did you find the experiment's task [a 5-point scale, from very easy to very difficult]? (4) To learn/follow the routes, did you find yourself using any particular strategies? (5) How did you remember which way to turn? (6) How did you remember where to turn? and (7) Please draw a map of the test route. The questionnaire used in Experiment 2 had two additional questions: Which pictures did you see in the test environment [participants were shown 16 pictures; 8 had been local landmarks in the test environment, and 8 had not been in any of the environments]?, and In what order did you pass those pictures (with respect to last route traversal)? These questions were added because, unlike in Experiment 1, every participant performed the test in an environment that contained local landmarks.

\section{Experiment 1}

Method

Participants Sixty-three individuals (30 men, 33 women) with a mean age of 27 years $(S D=7.4)$ took part. Six participants withdrew from the study because of motion sickness caused by traveling through a richly textured VE while physically stationary, and 1 was discarded (see the Results for this experiment). The remaining 56 participants were randomly assigned to each group (None, Local, Global, or Both landmarks), subject to there being equal numbers (7) of men and women in each group.

In the practice task and test, the None group used VEs that contained no supplementary landmarks (see Fig. 2a), the Local group used VEs that only contained local landmarks (see Fig. 2b), the Global group used VEs that only contained global landmarks (see Fig. 2c), and the Both group used VEs that contained both local and global landmarks (see Fig. 2d). In each group, half of the participants used each of the test routes.

Procedure and materials The procedure, hardware, and software were as described above. The laptop was connected to a 20 -in. Dell flat panel display $(1,600 \times 1,200$ pixels $)$, and the graphical field of view was $49^{\circ} \times 38^{\circ}$. All participants used the Rumblepad joysticks to travel through the VEs, meaning that they were only provided with visual navigational cues.

\section{Results}

The data for 1 participant in the Local group were discarded, because this person made a very large number of errors $(105 \%$ more than the next worst in the same group, $21 \%$ more than any other participant in the experiment), $30 \%$ of which occurred while traveling backward or sideways (indicative of performing the task by trial and error, rather than by trying to learn the route). The remainder of this section reports the test route data for the 56 remaining participants.

Four types of data were analyzed: the number of errors participants made, the type of the first error (if any) that was made at each decision point, where participants looked, and the number of route segments that participants drew on their sketch map. The sketch map data were analyzed using a two-factor (Local $\times$ Global Landmarks) ANOVA. The other data were analyzed using mixed factorial ANOVAs that had Local $\times$ Global Landmarks as between-participants factors, Journey $\times$ Trip Direction as within-participants factors, and, for the type of first error, Error Type as a third within-participants factor. Only the data for Journeys 2-4 were analyzed, meaning that participants had already made a guided trip in the outward direction and an unguided trip in the return direction. For clarity, only significant main effects and interactions are reported. $\mathrm{A}^{\dagger}$ after a $p$ value indicates that the Greenhouse-Geisser correction was applied, because the Mauchly sphericity test was significant. 
Number of errors An ANOVA showed that participants made significantly fewer errors with local landmarks, $F(1,52)=4.10, M S E=93.09, p=.05, \eta_{\mathrm{p}}^{2}=.07$; as the journeys progressed, $F(2,104)=8.46, M S E=6.02, p=$ $.001, \eta_{\mathrm{p}}^{2}=.14$; and in the outward direction, $F(1,52)=$ $11.41, M S E=10.02, p=.001, \eta_{\mathrm{p}}^{2}=.18$. There was also a significant interaction between direction, local landmarks, and global landmarks, $F(1,52)=8.58, M S E=10.02$, $p=.005, \eta_{\mathrm{p}}^{2}=.14$ (see Fig. 3 ).

The questionnaire indicated that only 6 participants played computer games frequently (at least once a week), and no group contained more than 2 of these participants. In terms of total errors, they were ranked within the best 15 of all the participants who took part.

To place the error data in context, simulation software was written to calculate the number of errors that would have been made if a navigator chose where to travel according to simple rules (an "ideal navigator"; Stankiewicz, Legge, Mansfield, \& Schlicht 2006). One million simulated route traversals were made, and the navigator always started in the correct direction (as participants had in the experiment), meaning that 14 decision points had to be negotiated. Successive choices were not allowed to be the same, but repeat errors could be made (e.g., left, then right, then left again). If the navigator chose at random between three directions of travel at each decision point (forward, left, or right), an average of 18.7 errors were made. However, if the navigator's first decision at each point was always to travel straight on, the average number of errors reduced to 12.0 , which is comparable with the None group on the first return trip.

Types of errors Participants made three types of errors: (i) traveling straight on where they should have turned, (ii) turning where they should have continued straight, and (iii)

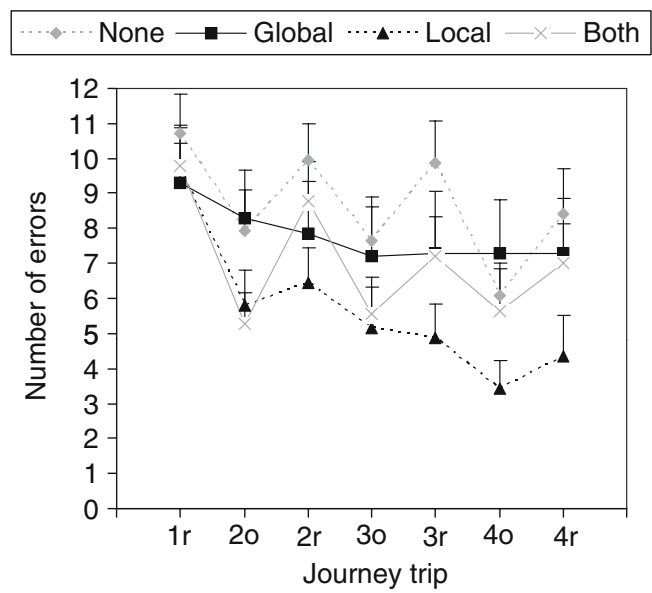

Fig. 3 Mean numbers of errors made by each group in Experiment 1. Journey is represented by the numbers $1-4$ and trip by o (outward) or $r$ (return). The outward trip of Journey 1 was guided. Error bars show standard errors of the means turning in the correct place but the wrong way. Postprocessing software was used to classify the first error that participants made at each decision point (see Table 1).

On any trip, up to eight errors of type (i) or (iii) above could be made, but only six of type (ii). The number of errors was converted to a percentage of the maximum possible number, and an ANOVA showed a main effect of error type, $F(2,64)=35.34, M S E=3187.68, p<.001^{\dagger}$, $\eta_{\mathrm{p}}^{2}=.40$, because participants traveled straight on where they should have turned $(37 \%)$ much more often than they either turned where they should have continued straight $(12 \%)$ or turned the wrong way $(11 \%)$. Participants made fewer errors with local landmarks, $F(1,52)=5.82, M S E=$ $1709.03, p=.02, \eta_{\mathrm{p}}^{2}=.10$; as the journeys progressed, $F(2,104)=8.26, M S E=121.71, p<.001, \eta_{\mathrm{p}}^{2}=.14$; and in the outward direction, $F(1,52)=12.17, M S E=210.79$, $p=.001, \eta_{\mathrm{p}}^{2}=.19$. There were also significant interactions for Direction $\times$ Error Type, $F(2,87)=4.60, M S E=228.67$, $p=.02^{\dagger}, \eta_{\mathrm{p}}^{2}=.08$, and Direction $\times$ Local Landmarks $\times$ Global Landmarks, $F(1,52)=11.55, M S E=210.79, p=$ $.001, \eta_{\mathrm{p}}^{2}=.18$. Note that, if a participant chose at random to travel straight, turn left, or turn right, then at a turn the chances of errors of types (i) and (iii) above were both $33 \%$, whereas at a straight-on decision point the chance of a type (ii) error was $67 \%$. If the above analysis had used these percentages, rather than the maximum possible number of each error type, the dominance of type (i) errors would have been strengthened.

Where participants looked To investigate the information used during route navigation, we analyzed where participants looked at the decision points where they did not make an error in a given traversal. From a participant's view direction while "at" each decision point (a $0.75 \mathrm{~m} \times$ $0.75 \mathrm{~m}$ area, the corridor intersection), postprocessing software determined whether the participant had just looked along the route or had looked around. From these data, the percentage of error-free decision points where participants looked around was calculated. For a straighton decision point, participants were considered to have looked around if they looked more than $45^{\circ}$ to the left or to the right of the route. For a turn, looking around occurred if a participant looked outside the $180^{\circ}$ arc that extended from $45^{\circ}$ on one side of straight ahead to $45^{\circ}$ on the other side of the turn. The $45^{\circ}$ criterion meant that participants' direction of view was divided into quadrants, which was appropriate, since the marketplace's layout was orthogonal.

An ANOVA showed that participants looked around significantly more when they were provided with local landmarks, $F(1,52)=5.01, M S E=733, p=.03, \eta_{\mathrm{p}}^{2}=.09$, and on return trips, $F(1,52)=18.66, M S E=132, p<.001$, $\eta_{\mathrm{p}}^{2}=.26$. However, overall, looking around only occurred 
Table 1 Types of errors made by each group in Experiment 1

\begin{tabular}{|c|c|c|c|c|c|c|c|c|c|c|}
\hline \multirow[t]{2}{*}{ Journey } & \multirow[t]{2}{*}{ Trip } & \multirow[t]{2}{*}{ Error type } & \multicolumn{2}{|c|}{ None } & \multicolumn{2}{|c|}{ Global } & \multicolumn{2}{|c|}{ Local } & \multicolumn{2}{|c|}{ Both } \\
\hline & & & $M$ & $S D$ & $M$ & $S D$ & $M$ & $S D$ & $M$ & $S D$ \\
\hline \multirow[t]{6}{*}{2} & \multirow[t]{3}{*}{ Outward } & Went straight on & 2.8 & 2.4 & 3.3 & 2.4 & 2.4 & 1.5 & 2.9 & 1.8 \\
\hline & & Turned & 0.9 & 1.6 & 1.0 & 1.3 & 0.6 & 0.8 & 0.6 & 1.0 \\
\hline & & Turned wrong way & 1.6 & 1.5 & 1.1 & 1.2 & 0.5 & 0.9 & 0.4 & 0.7 \\
\hline & \multirow[t]{3}{*}{ Return } & Went straight on & 3.6 & 2.5 & 3.4 & 2.6 & 3.3 & 1.8 & 4.0 & 2.3 \\
\hline & & Turned & 1.7 & 1.9 & 0.6 & 0.7 & 0.4 & 0.6 & 0.7 & 0.7 \\
\hline & & Turned wrong way & 1.4 & 1.2 & 0.9 & 0.9 & 0.4 & 0.6 & 1.1 & 0.9 \\
\hline \multirow[t]{6}{*}{3} & \multirow[t]{3}{*}{ Outward } & Went straight on & 2.7 & 2.2 & 3.4 & 2.3 & 2.1 & 2.0 & 2.4 & 2.0 \\
\hline & & Turned & 1.0 & 1.9 & 0.6 & 0.8 & 0.5 & 0.7 & 0.6 & 0.9 \\
\hline & & Turned wrong way & 1.4 & 1.0 & 0.9 & 1.1 & 0.6 & 0.8 & 0.8 & 1.1 \\
\hline & \multirow[t]{3}{*}{ Return } & Went straight on & 3.4 & 2.9 & 3.1 & 2.3 & 2.7 & 2.2 & 3.5 & 2.4 \\
\hline & & Turned & 1.6 & 2.0 & 0.4 & 0.6 & 0.3 & 0.5 & 0.5 & 0.7 \\
\hline & & Turned wrong way & 1.6 & 1.3 & 0.9 & 1.0 & 0.5 & 0.9 & 0.9 & 1.1 \\
\hline \multirow[t]{6}{*}{4} & \multirow[t]{3}{*}{ Outward } & Went straight on & 2.5 & 2.4 & 3.3 & 2.6 & 1.4 & 1.1 & 2.7 & 2.3 \\
\hline & & Turned & 1.1 & 1.0 & 0.6 & 0.9 & 0.6 & 0.8 & 0.6 & 0.9 \\
\hline & & Turned wrong way & 0.9 & 1.0 & 0.7 & 1.3 & 0.4 & 0.6 & 0.4 & 0.6 \\
\hline & \multirow[t]{3}{*}{ Return } & Went straight on & 3.1 & 2.8 & 3.1 & 2.6 & 1.9 & 1.8 & 3.4 & 2.6 \\
\hline & & Turned & 1.4 & 1.6 & 0.5 & 0.5 & 0.6 & 1.0 & 0.6 & 0.6 \\
\hline & & Turned wrong way & 1.6 & 1.6 & 0.7 & 0.8 & 0.4 & 0.8 & 0.6 & 1.0 \\
\hline
\end{tabular}

On each trip, the sum of all error types equals the number of decision points at which an error was made made and that the effect of the two types of landmarks would be complementary. Local landmarks did reduce participants' errors, a finding that is consistent with previous research in which participants learned a route in one direction (Jansen-Osmann \& Fuchs, 2006) and that adds to a growing body of evidence suggesting that landmarks primarily facilitate traveling between specific places rather than learning the overall layout of a space (Ruddle, 2005). Local landmarks primarily provided positional information, which directly addressed the most common type of error that participants made (incorrectly traveling straight on), although it should be noted that there was no interaction between error type and landmarks.

Contrary to our hypothesis, global landmarks did not affect the overall number of errors that participants made. Previous research has suggested that global landmarks only have an effect for certain navigational tasks (Evans et al.,

1984; Ruddle \& Lessels, 2009). One reason for the lack of
We hypothesized that both local and global landmarks would reduce the overall number of errors that participants
Table 2 Percentages of errorfree decision points where participants looked around in Experiment 1

\begin{tabular}{|c|c|c|c|c|c|c|c|c|c|}
\hline \multirow[t]{2}{*}{ Journey } & \multirow[t]{2}{*}{ Trip } & \multicolumn{2}{|c|}{ None } & \multicolumn{2}{|c|}{ Global } & \multicolumn{2}{|c|}{ Local } & \multicolumn{2}{|c|}{ Both } \\
\hline & & $M$ & $S D$ & $M$ & $S D$ & $M$ & $S D$ & $M$ & $S D$ \\
\hline \multirow[t]{2}{*}{2} & Outward & 2.5 & 5.4 & 5.5 & 8.0 & 13.6 & 14.3 & 12.0 & 11.2 \\
\hline & Return & 5.9 & 7.4 & 11.1 & 18.1 & 19.6 & 17.9 & 15.1 & 16.6 \\
\hline \multirow[t]{2}{*}{3} & Outward & 3.0 & 7.0 & 9.3 & 14.4 & 5.6 & 10.2 & 12.6 & 14.4 \\
\hline & Return & 8.2 & 8.4 & 8.6 & 16.0 & 18.1 & 18.9 & 18.0 & 21.2 \\
\hline \multirow[t]{2}{*}{4} & Outward & 5.5 & 9.8 & 7.2 & 8.9 & 10.0 & 12.2 & 5.4 & 10.1 \\
\hline & Return & 6.8 & 12.4 & 11.5 & 15.2 & 23.3 & 20.1 & 10.8 & 17.1 \\
\hline
\end{tabular}


an effect in the present study is that only one of the less common types of error (iii) benefited from the orientation information that global landmarks provide. However, the lack of an effect may also have been influenced by the orthogonal structure of the environment, which only offered three choices at decision points (turn left/right or continue straight), which participants may have memorized using a verbal strategy. Related to this, it is worth noting that the error type and looking-around data suggest that, if participants made a correct turn, they did so by recalling the correct direction (e.g., turn left; a landmark-action pair) rather than by extracting directional cues from the landmarks by looking around at each decision point.

The main effects of journey and direction on the numbers of errors that occurred showed that participants learned the route but had worse knowledge about how to travel back than in the outward direction. However, there was also a complex interaction (Direction $\times$ Local Landmarks $\times$ Global Landmarks). The Global group improved gradually and, as hypothesized, used knowledge gained when traversing the route in one direction to help traverse it in the other. By contrast, the None and Both groups made substantially more errors on the return trips than on the outward trips. It should also be noted that, on return trips, the Both group's performance deteriorated to the level of the Global group, suggesting that local landmarks were used less than global landmarks because the latter were visible from more places and for longer.

Finally, referring again to the error type data, most of the additional errors that the Local and Both groups made on the return trips, relative to on the outward trips, were due to not knowing where to turn. However, all three types of errors were more common on the return trips for the None group, whereas the Global group's errors showed little change.

\section{Experiment 2}

This experiment investigated the effect of translational body-based information on route knowledge. One group of participants (the Walk group) was provided with bodybased information for both components of movement (translation and rotation), whereas the other, Rotate group only had body-based information for rotational movement. Both groups used the local landmark VEs from Experiment 1, because that was the type of environment in which participants had made the fewest errors.

\section{Method}

Participants Forty-four individuals (20 men, 24 women) with a mean age of 25 years $(S D=4.5)$ took part. Six participants withdrew from the study because of motion sickness, and 2 were discarded (see the Results below). The remaining participants were randomly assigned to each group (Rotate or Walk), subject to there being equal numbers (9) of men and women in each group.

Materials Details of the VEs, hardware, and software are described above (see the Overview of Experiments section). The laptop was connected to an nVisor SX HMD via a Matrox DualHead2Go video splitter, giving a stereo view and $47^{\circ} \times 38^{\circ}(1,280 \times 1,024$ pixels $)$ in each eye.

Procedure The procedure was the same as in Experiment 1, except that the first journey of the interface practice route was performed using a monitor display (as in Experiment 1), and the other journeys used the HMD (walking or physically rotating, depending on a participant's group). Participants listened to white noise in headphones to mask any auditory orientation cues from the tracking hall (these were unlikely to have occurred, because the hall was empty and silent), and the Walk group were blindfolded while entering and leaving the hall so that they could not use knowledge of the general size of the hall to help memorize the route. The questionnaire had two additional questions, which related to the local landmarks (see the Overview of Experiments).

\section{Results}

The data for 2 participants in the Rotate group were discarded because they made more than $90 \%$ more errors than anyone else in the experiment. The remainder of this section reports the test route data for the 36 remaining participants ( 9 men and 9 women in each group).

The results for Journeys 2-4 were analyzed using the same types of ANOVA as in Experiment 1, except that there was only one between-participants factor (Group). For clarity, only significant main effects and interactions are reported. $\mathrm{A}^{\dagger}$ after a $p$ value indicates that the GreenhouseGeisser correction was applied.

Number of errors An ANOVA showed that the Walk group made significantly fewer errors than the Rotate group, $F(1,34)=4.62, M S E=40.85, p=.04, \eta_{\mathrm{p}}^{2}=.12$, and that participants made fewer errors as the journeys progressed, $F(2,68)=8.78, M S E=5.37, p<.001, \eta_{\mathrm{p}}^{2}=.20$, and in the outward direction, $F(1,34)=5.88, M S E=10.24, p=.02$, $\eta_{\mathrm{p}}^{2}=.15$. There was also a significant Group $\times$ Direction interaction, $F(1,34)=5.67, M S E=10.24, p=.02$, $\eta_{\mathrm{p}}^{2}=.14$. It is also worth noting that, unlike local landmarks (see Fig. 3), full body-based information also provided an advantage on the first return trip as compared with the Rotate group (see Fig. 4), and a one-way ANOVA showed that the difference was significant, $F(1,34)=5.83$, 


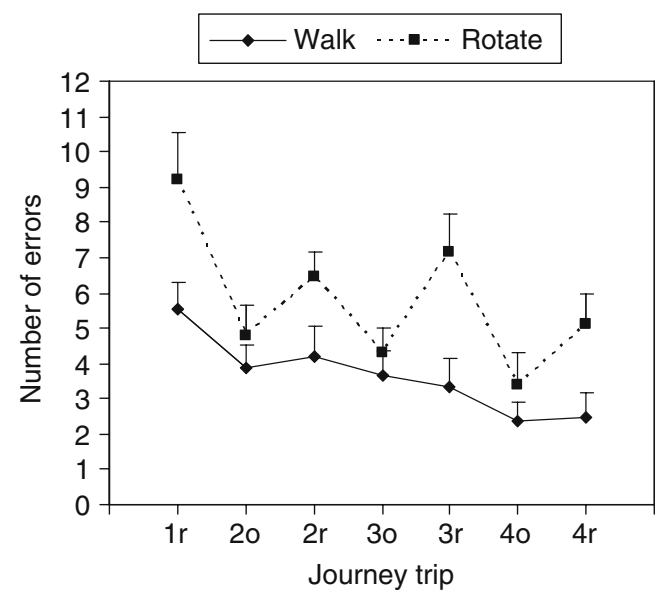

Fig. 4 Mean numbers of errors made by each group in Experiment 2. Journey is represented by the numbers $1-4$ and trip by o (outward) or $r$ (return). The outward trip of Journey 1 was guided. Error bars show standard errors of the means

$M S E=20.75, p=.02, \eta_{\mathrm{p}}^{2}=.15$. Averaged across all the sessions, the Walk group made 36\% fewer errors than the Rotate group.

The joystick allowed the Rotate group to move in any direction relative to their direction of view, which meant that some errors occurred when participants moved sideways or diagonally or "stepped" backwards. On some of those occasions, the root cause of the error may have had more to do with a lack of concentration when controlling the interface than with a genuine route-finding error. To investigate this, the numbers of errors that participants made simply while moving forward was analyzed, even though this was likely to underestimate the true number of errors. This showed the same pattern of results as the full error data above, except that there was no main effect of group (the two groups made similar numbers of errors on the outward trips, but the Rotate group made 57\% more errors on the return trips than the Walk group).

The questionnaire data indicated that only 5 of the participants (4 of these were in the Rotate group) played computer games at least once a week. These 5 were ranked from second to twenty-fifth in terms of the number of errors made.

Types of errors As well as the three types of errors that participants made in Experiment 1 (traveling straight on where they should have turned, turning the wrong way, and turning where they should have continued straight), on a small number of occasions participants here tried to move backward along the route (see Table 3). The software prevented this, and that category of "accidental" error was excluded from the following analysis.

As in Experiment 1, the number of errors was converted to a percentage of the maximum possible number, and an ANOVA showed a main effect of error type, $F(2,42)=$
Table 3 Types of errors made by each group in Experiment 2

\begin{tabular}{|c|c|c|c|c|c|c|}
\hline \multirow[t]{2}{*}{ Journey } & \multirow[t]{2}{*}{ Trip } & \multirow[t]{2}{*}{ Error type } & \multicolumn{2}{|c|}{ Walk } & \multicolumn{2}{|c|}{ Rotate } \\
\hline & & & $M$ & $S D$ & $M$ & $S D$ \\
\hline \multirow[t]{8}{*}{2} & \multirow[t]{4}{*}{ Outward } & Went straight on & 1.5 & 1.3 & 2.3 & 1.7 \\
\hline & & Turned & 0.5 & 0.6 & 0.3 & 0.7 \\
\hline & & Turned wrong way & 0.7 & 0.8 & 0.5 & 0.7 \\
\hline & & Went backward & 0.1 & 0.3 & 0.1 & 0.3 \\
\hline & \multirow[t]{4}{*}{ Return } & Went straight on & 1.6 & 1.9 & 2.7 & 1.8 \\
\hline & & Turned & 0.7 & 0.8 & 0.7 & 0.8 \\
\hline & & Turned wrong way & 0.6 & 0.9 & 0.7 & 0.8 \\
\hline & & Went backward & 0.1 & 0.3 & 0.1 & 0.2 \\
\hline \multirow[t]{8}{*}{3} & \multirow[t]{4}{*}{ Outward } & Went straight on & 1.6 & 1.3 & 1.7 & 1.4 \\
\hline & & Turned & 0.4 & 0.7 & 0.3 & 0.7 \\
\hline & & Turned wrong way & 0.4 & 0.6 & 0.7 & 0.8 \\
\hline & & Went backward & 0.1 & 0.3 & 0.2 & 0.5 \\
\hline & \multirow[t]{4}{*}{ Return } & Went straight on & 1.6 & 1.6 & 2.9 & 1.5 \\
\hline & & Turned & 0.4 & 0.7 & 0.5 & 0.6 \\
\hline & & Turned wrong way & 0.3 & 0.6 & 0.6 & 0.9 \\
\hline & & Went backward & 0.1 & 0.3 & 0.1 & 0.3 \\
\hline \multirow[t]{8}{*}{4} & \multirow[t]{4}{*}{ Outward } & Went straight on & 1.1 & 1.3 & 1.4 & 1.4 \\
\hline & & Turned & 0.3 & 1.0 & 0.2 & 0.5 \\
\hline & & Turned wrong way & 0.4 & 0.6 & 0.5 & 0.7 \\
\hline & & Went backward & 0.1 & 0.2 & 0.1 & 0.3 \\
\hline & \multirow[t]{4}{*}{ Return } & Went straight on & 1.1 & 1.3 & 1.9 & 1.3 \\
\hline & & Turned & 0.4 & 0.6 & 0.2 & 0.6 \\
\hline & & Turned wrong way & 0.5 & 0.9 & 0.9 & 1.0 \\
\hline & & Went backward & 0.1 & 0.2 & 0.2 & 0.4 \\
\hline
\end{tabular}

On each trip, the sum of all error types equals the number of decision points at which an error was made

36.50, MSE $=754.14, p<.001^{\dagger}, \eta_{\mathrm{p}}^{2}=.52$, because participants traveled straight on where they should have turned (22\%) much more often than the other types of errors (both 7\%). Participants also made fewer errors as the journeys progressed, $F(2,57)=7.64, M S E=164.35, p=$ $.002^{\dagger}, \eta_{\mathrm{p}}^{2}=.18$, and in the outward direction, $F(1,34)=$ 8.96, $M S E=131.82, p=.005, \eta_{\mathrm{p}}^{2}=.21$. There were also three significant interactions. A Group $\times$ Direction interaction occurred because the Rotate group made errors at substantially more decision points on the return trips, whereas the Walk group was largely unaffected by the trip direction, $F(1,34)=7.49, M S E=131.82, p=.01$, $\eta_{\mathrm{p}}^{2}=.18$. An Error Type $\times$ Journey interaction occurred because the proportions of errors in which participants turned the wrong way remained constant, but the other types of errors decreased as the journeys progressed, $F(4,136)=3.25, M S E=103.48, p=.01, \eta_{\mathrm{p}}^{2}=.09$. An Error Type $\times$ Group interaction occurred because the Rotate group traveled straight on where they should have turned more often than the Walk group did, $F(2,68)=3.88, M S E=$ $754.14, p=.02, \eta_{\mathrm{p}}^{2}=.10$. 
Where participants looked In the analysis of where participants looked, there were no significant effects or interactions. Overall, the Walk group looked around at $18.3 \%$ of the decision points where a participant did not make an error, and the Rotate group looked around at $10.5 \%$ (see Table 4).

Sketch maps and picture tests The sketch maps were scored in the same way as in Experiment 1, with 1 participant excluded because their sketch contained a gap. The Walk group's map error was smaller then the Rotate group's $(M=$ $1.2, S D=1.7$, vs. $M=2.1, S D=2.1$ ), but an ANOVA showed that the difference was not significant, $F(1,34)=$ 1.62, MSE $=3.64, p=.21, \eta_{\mathrm{p}}^{2}=.05$, and there was not a significant correlation between the sketch map and routefinding errors, $r(35)=.06, p>.05$.

Picture recognition was scored by giving one mark for each picture that a participant correctly stated had been in the environment and subtracting one for identified pictures that had not (maximum score $=8$ ). The Walk group recognized significantly more pictures $(M=6.4, S D=1.1)$ than the Rotate group $(M=5.6, S D=1.5), F(1,35)=3.97, M S E=$ $1.79, p=.05, \eta_{\mathrm{p}}^{2}=.10$. Picture order along the route was scored by first giving one mark for each picture in a participant's order and then using string matching to subtract a mark for each operation (insertion, deletion, or edit) that was required to change the order into the correct one (Levenshtein, 1966), and again, the maximum possible score was 8 . One participant in the Walk group was omitted for not providing an answer. The Walk group's picture order was significantly more accurate $(M=2.8, S D=1.0)$ than the Rotate group's $(M=1.6, S D=1.1), F(1,34)=12.20, M S E=1.05, p=.001$, $\eta_{\mathrm{p}}^{2}=.27$.

\section{Discussion}

In every traversal, the Walk group made fewer errors than the Rotate group. The overall advantage provided to the Walk group by translational body-based information was predicted, but the more pronounced advantage on return

Table 4 Percentages of error-free decision points where participants looked around in Experiment 2

\begin{tabular}{|c|c|c|c|c|c|}
\hline \multirow[t]{2}{*}{ Journey } & \multirow[t]{2}{*}{ Trip } & \multicolumn{2}{|l|}{ Walk } & \multicolumn{2}{|c|}{ Rotate } \\
\hline & & $M$ & $S D$ & $M$ & $S D$ \\
\hline \multirow[t]{2}{*}{2} & Outward & 20.6 & 22.1 & 9.2 & 16.2 \\
\hline & Return & 21.4 & 18.1 & 11.4 & 18.4 \\
\hline \multirow[t]{2}{*}{3} & Outward & 17.7 & 17.1 & 10.1 & 13.2 \\
\hline & Return & 21.3 & 22.2 & 11.7 & 13.4 \\
\hline \multirow[t]{2}{*}{4} & Outward & 11.5 & 12.0 & 9.4 & 11.1 \\
\hline & Return & 17.4 & 14.2 & 11.0 & 9.5 \\
\hline
\end{tabular}

trips was not. That advantage arose because the translational information helped the Walk group remember where to turn for both trip directions, but the Rotate group were less knowledgeable about where to turn on return legs. In both groups, if participants made the correct navigational decision, they did so without looking around, indicating that if participants knew where to turn they could also remember which way to turn.

The advantage provided by translational body-based information is consistent with some previous research (Ruddle \& Lessels, 2009), but it contrasts with the findings of studies that have used triangle completion tasks (Avraamides et al., 2004; Klatzky et al., 1998) or asked participants to memorize a setting in a room and then point to objects or identify changes (May, 2004; Rieser, 1989; Simons \& Wang, 1998). The differences between these studies' findings may be explained by considering the complexity of the tasks that participants performed. Rotational body-based information seems to be required for basic spatial tasks, whereas additional information about how far one has traveled (i.e., translational body-based information) is required for tasks that involve higher-level cognitive processes such as planning where to travel. Intermediate tasks, such as memorizing the locations of objects along a prescribed path, are at a crossover point in terms of cognitive load, which would explain why studies using such tasks show a gradual performance improvement as more components of body-based information are provided (Chance et al., 1998).

Participants' accuracy for recognizing and ordering the pictures in the present study was substantially lower than in Ishikawa and Montello (2006), but in that study participants were driven along a route in a car and explicitly told to memorize four particular landmarks. It is also worth noting that that study found no difference between participants who only traversed a route in one direction, compared with those who made traversals in both directions.

\section{General discussion}

The primary aim of this study was to investigate the contributions that global versus local landmarks and the translational component of body-based information make to route knowledge. This was achieved by measuring both the quantity and type of participants' errors and by analyzing their navigational behavior. In terms of ecological validity, this study stands out because it is one of very few that have investigated the everyday task of navigating a route both to and from a given location. The present study also used much richer visual scenes than have appeared in most previous laboratory-based studies, albeit one that was still less rich than most real-world environments. Against this, it 
should be noted that, although the environment was large scale (participants had to travel through it to resolve the necessary navigational detail), it was small in extent because it had to fit within a $13 \times 12 \mathrm{~m}$ tracking hall so that the effect of translational body-based information could be studied.

When only visual information was provided (Experiment 1), local landmarks were beneficial to route knowledge, and this was consistent with our predictions. However, contrary to our predictions, global landmarks did not improve performance. An explanation comes from the error type data, which showed that participants were less sure of where to turn than which way. However, it should also be noted that the orthogonal grid structure of the environment may have encouraged participants to memorize turns as left or right, rather than toward $\mathrm{X}$ or $\mathrm{Y}$, even though the performance of the None group shows that that structure did not trivialize participants' task.

Experiment 2 showed that providing both components (translation and rotation) of body-based information, rather than just the rotational component, led to participants making $36 \%$ fewer errors. No previous research has demonstrated this for route navigation, but the results are consistent with those when a different task (navigational search) was conducted in a small-scale space (Ruddle \& Lessels, 2009) and indicate that translational body-based information becomes more important as task complexity increases. As with global landmarks, the environment's orthogonal structure may have reduced the contribution that rotational body-based information made to participants' route knowledge. However, previous research showed that rotational information did not improve participants' navigational performance in orthogonally or obliquely structured environments (Ruddle et al., 1999; Ruddle \& Péruch, 2004).

A notable finding was that trip direction affected participants' performance in some conditions but not others. In particular, and contrary to our predictions, local and global landmarks interfered with each other, so participants who were provided with both made more errors on return trips than did participants who only had local landmarks. An explanation is provided by saliency, because the global landmarks were more visible than local landmarks, but participants performed better when they were forced to rely on the latter. Of the two groups who were least affected by trip direction, the Global group were provided with allocentric landmark cues, whereas the Walk group could complement external cues with internal sensory information about how far and in which direction they had turned. This may have helped participants form a single, integrated representation of the route, or may have just reduced errors made when mentally transforming route knowledge gained when traveling in one direction to another direction. The strong effect of trip direction found in the present study raises questions about previous studies that have investigated landmark memory. For example, would the pattern of results have been different for landmark recognition and priming if out-and-back, instead of one-way, journeys had been made (e.g., Jansen-Osmann \& Wiedenbauer, 2004; Janzen, 2006; Janzen \& van Turennout, 2004)?

In the present study, the error type data showed that participants were much less sure of where to turn (i.e., errors in which participants incorrectly went straight on or turned instead of continuing straight; see Tables 1 and 3) than of which way to turn. In addition, most of the differences between the groups occurred when participants incorrectly traveled straight on. This is consistent with the types of information that caused the principal performance differences in our two experiments, because local landmarks allowed participants to recognize the places to turn, and the translational component of body-based information helped the Walk group code the length of each route segment and, therefore, correctly recall where they should turn. Previous research has suggested that, when in doubt, people tend to travel straight on (Meilinger, Franz, \& Bülthoff, in press), and this is supported by the results of the present study. Our results also place in context the findings from studies that used linear routes, where participants only had to choose at each decision point whether to travel through a door on the left or the right (Tlauka \& Wilson, 1994; Waller \& Lippa, 2007). That equates to the type (iii) errors in the present experiments (turning in the correct place but the wrong way), which only accounted for a minority of participants' route-finding errors.

In terms of generalization, key factors to consider are how our findings would apply to environments that either have a less regular structure (e.g., oblique intersections) or allow unrestricted movement. Many built environments (e.g., buildings and towns) and natural environments (e.g., forests or mountain ranges) have somewhat irregular structures but still involve navigation along defined corridors/pavements/paths from one decision point to the next. At each decision point, the navigator chooses between a discrete set of options, for which only gross orientation information is required. In these environments, translational decisions also only need to be accurate in a gross sense, because the navigator will turn when encountering a decision point that is in approximately the correct place (e.g., they may see that the next decision point is clearly too far). This lessens the impact of errors that accumulate during path integration, strengthening the likely importance of body-based information, while global landmarks are likely to play an enhanced role if the intersections are oblique. The situation in environments that allow unrestricted movement (e.g., an open plane) is less clear. Global 
landmarks will be important because humans' internal orientation cues are not always reliable (Souman, Frissen, Sreenivasa, \& Ernst 2009), and translational body-based information may retain its importance to calibrate movement within the environment.

Finally, from an applied perspective, the small number of errors made by the Walk group, relative to the Rotate group, indicates that VEs would become much more effective for many spatial applications if physical locomotion devices such as omnidirectional treadmills were to be perfected (Darken, Cockayne, \& Carmein 1997; De Luca, Mattone, Giordano, \& Bülthoff 2009). Until now, the likely benefit of such devices has not been demonstrated.

\section{References}

Avraamides, M. N., Klatzky, R. L., Loomis, J. M., \& Golledge, R. G. (2004). Use of cognitive versus perceptual heading during imagined locomotion depends on the response mode. Psychological Science, 15, 403-408.

Chance, S. S., Gaunet, F., Beall, A. C., \& Loomis, J. M. (1998). Locomotion mode affects the updating of objects encountered during travel: the contribution of vestibular and proprioceptive inputs to path integration. Presence: Teleoperators and Virtual Environments, 7, 168-178.

Cornell, E. H., Heth, C. D., \& Rowat, W. L. (1992). Wayfinding by children and adults: response to instructions to use look-back and retrace strategies. Developmental Psychology, 28, 328-336.

Darken, R., Cockayne, W., \& Carmein, D. (1997). The omnidirectional treadmill: a locomotion device for virtual worlds. In Proceedings of the 10th Annual ACM Symposium on User Interface Software and Technology (pp. 213-221). New York: ACM Press.

De Luca, A., Mattone, R., Giordano, P. R., \& Bülthoff, H. H. (2009). Control design and experimental evaluation of the 2D CyberWalk platform. In Proceedings of the IEEE/RSJ International Conference on Intelligent Robots and Systems (IROS 2009) (pp. 5051-5058). Los Alamitos, CA: IEEE Press.

Denis, M. (1997). The description of routes: a cognitive approach to the production of spatial discourse. Current Psychology of Cognition, 16, 409-458.

Evans, G. W., Skorpanich, M. A., Gärling, T., Bryant, K. J., \& Bresolin, B. (1984). The effects of pathway configuration, landmarks and stress on environmental cognition. Journal of Environmental Psychology, 4, 323-335.

Farrell, M., Arnold, P., Pettifer, S., Adams, J., Graham, T., \& MacManamon, M. (2003). Transfer of route learning from virtual to real environments. Journal of Experimental Psychology. Applied, 9, 219-227.

Foo, P., Warren, W. H., Duchon, A., \& Tarr, M. J. (2005). Do humans integrate routes into a cognitive map? Map- versus landmarkbased navigation of novel shortcuts. Journal of Experimental Psychology. Learning, Memory, and Cognition, 31, 195-215.

Gillner, S., Weiß, A. M., \& Mallot, H. A. (2008). Visual homing in the absence of feature-based landmark information. Cognition, 109, $105-122$.

Hurlebaus, R., Basten, K., Mallot, H. A., \& Wiener, J. M. (2008). Route learning strategies in a virtual cluttered environment. In C. Freksa (Ed.), Spatial cognition VI: Learning, reasoning and talking about space. Berlin: Springer. Lecture Notes in Computer Science, Vol. 5248, pp. 104-120.

Ishikawa, T., \& Montello, D. (2006). Spatial knowledge acquisition from direct experience in the environment: individual differences in the development of metric knowledge and the integration of separately learned places. Cognitive Psychology, 52, 93-129.

Jansen-Osmann, P., \& Fuchs, P. (2006). Wayfinding behavior and spatial knowledge of adults and children in a virtual environment: the role of landmarks. Experimental Psychology, 5, 171-181.

Jansen-Osmann, P., \& Wiedenbauer, G. (2004). The representation of landmarks and routes in children and adults: a study in a virtual environment. Journal of Environmental Psychology, 24, 347-357.

Janzen, G. (2006). Memory for object location and route direction in virtual large-scale space. The Quarterly Journal of Experimental Psychology, 59, 493-508.

Janzen, G., \& van Turennout, M. (2004). Selective neural representation of objects relevant for navigation. Nature Neuroscience, 7 , 673-677.

Klatzky, R. L., Loomis, J. M., Beall, A. C., Chance, S. S., \& Golledge, R. G. (1998). Spatial updating of self-position and orientation during real, imagined, and virtual locomotion. Psychological Science, 9, 293-298.

Levenshtein, V. (1966). Binary codes capable of correcting deletions, insertions and reversals. Soviet Physics Doklady, 10, 707-710.

Loomis, J., Klatzky, R. L., Golledge, R. G., \& Philbeck, J. W. (1999). Human navigation by path integration. In R. Golledge (Ed.), Wayfinding: Cognitive mapping and other spatial processes (pp. 125-151). Baltimore: John Hopkins University Press.

Lynch, K. (1960). The image of the city. Cambridge: MIT Press.

May, M. (2004). Imaginal perspective switches in remembered environments: transformation versus interference accounts. Cognitive Psychology, 48, 163-206.

Meilinger, T., Franz, G., \& Bülthoff, H. H. (in press). From isovists via mental representations to behaviour: first steps toward closing the causal chain. Environment and Planning B: Planning and Design. doi:10.1068/b34048t

Montello, D. R. (1998). A new framework for understanding the acquisition of spatial knowledge in large-scale environments. In R. Golledge \& M. Egenhofer (Eds.), Spatial and temporal reasoning in geographic information systems (pp. 143-154). New York: Oxford University Press.

National Travel Survey: Why People Travel. (2009). Retrieved 16 September, 2010, from www.dft.gov.uk/pgr/statistics/datatables publications/nts/

Rieser, J. (1989). Access to knowledge of spatial structure at novel points of observation. Journal of Experimental Psychology. Learning, Memory, and Cognition, 15, 1157-1165.

Ruddle, R. A. (2005). The effect of trails on first-time and subsequent navigation in a virtual environment. In Proceedings of the IEEE Virtual Reality Conference (pp. 115-122). Los Alamitos: IEEE Press.

Ruddle, R. A., \& Lessels, S. (2009). The benefits of using a walking interface to navigate virtual environments. ACM Transactions on Computer-Human Interaction, 16, 5.

Ruddle, R. A., Payne, S. J., \& Jones, D. M. (1997). Navigating buildings in "desk-top" virtual environments: experimental investigations using extended navigational experience. Journal of Experimental Psychology. Applied, 3, 143-159.

Ruddle, R. A., Payne, S. J., \& Jones, D. M. (1999). Navigating largescale virtual environments: what differences occur between helmet-mounted and desk-top displays? Presence: Teleoperators and Virtual Environments, 8, 157-168.

Ruddle, R. A., \& Péruch, P. (2004). Effects of proprioceptive feedback and environmental characteristics on spatial learning in virtual environments. International Journal of Human-Computer Studies, 60, 299-326. 
Siegel, A. W., \& White, S. H. (1975). The development of spatial representations of large scale environments. In H. W. Reese (Ed.), Advances in child development and behavior (pp. 9-55). New York: Academic Press.

Simons, D. J., \& Wang, R. F. (1998). Perceiving real-world viewpoint changes. Psychological Science, 9, 315-320.

Souman, J. L., Frissen, I., Sreenivasa, M. N., \& Ernst, M. O. (2009). Walking straight into circles. Current Biology, 19, 1538-1542.

Stankiewicz, B. J., \& Kalia, A. A. (2007). Acquisition of structural versus object landmark knowledge. Journal of Experimental Psychology: Human Perception and Performance, 33, 378-390.

Stankiewicz, B. J., Legge, G. E., Mansfield, J. S., \& Schlicht, E. J. (2006). Lost in virtual space: studies in human and ideal spatial navigation. Journal of Experimental Psychology: Human Perception and Performance, 32, 688-704.

Steck, S. D., \& Mallot, H. A. (2000). The role of global and local landmarks in virtual environment navigation. Presence: Teleoperators and Virtual Environments, 9, 69-83.

Tlauka, M., \& Wilson, P. N. (1994). The effects of landmarks on route-learning in a computer-simulated environment. Journal of Environmental Psychology, 14, 305-313.

Waller, D., \& Lippa, Y. (2007). Landmarks as beacons and associative cues: their role in route learning. Memory \& Cognition, 35, 910-924.

Williamson, J., \& Barrow, C. (1994). Errors in everyday routefinding: a classification of types and possible causes. Applied Cognitive Psychology, 8, 513-524. 